\title{
Proton pump inhibitors: use and misuse in the clinical setting.
}

\section{Vincenzo Savarino, Elisa Marabotto, Patrizia Zentilin, Manuele Furnari, Giorgia Bodini, Costanza De Maria, Gaia Pellegatta, Claudia Coppo \& Edoardo Savarino}

To cite this article: Vincenzo Savarino, Elisa Marabotto, Patrizia Zentilin, Manuele Furnari, Giorgia Bodini, Costanza De Maria, Gaia Pellegatta, Claudia Coppo \& Edoardo Savarino (2018): Proton pump inhibitors: use and misuse in the clinical setting., Expert Review of Clinical Pharmacology, DOI: $10.1080 / 17512433.2018 .1531703$

To link to this article: https://doi.org/10.1080/17512433.2018.1531703

Accepted author version posted online: 08

Oct 2018.

Submit your article to this journal $\sqsubset$

View Crossmark data \lceil 
Publisher: Taylor \& Francis

Journal: Expert Review of Clinical Pharmacology

DOI: $10.1080 / 17512433.2018 .1531703$

Review

Proton pump inhibitors : use and misuse in the clinical setting.

Vincenzo Savarino1, Elisa Marabotto1, Patrizia Zentilin1, Manuele Furnari1, Giorgia Bodini1, Costanza De Maria1, Gaia Pellegatta1, Claudia Coppo1 and Edoardo Savarino2.

1. Gastrointestinal Unit, Department of Internal Medicine, University of Genoa, Genoa, Italy

2. Gastrointestinal Unit, Department of Surgery, Oncology and Gastroenterology, University of Padua, Padua, Italy

Corresponding Author :

Vincenzo Savarino

Department of Internal Medicine, University of Genoa, Genoa, Italy Viale Benedetto XV, No. 6, 16132 Genoa (Italy)

Telephone : +390103538956

e-mail : vsavarin@unige.it

\section{Abstract}

Introduction: The introduction of proton pump inhibitors (PPIs) into clinical practice has greatly improved our therapeutic approach to acid-related diseases for their efficacy and safety.

Areas Covered:The following evidence-based indications for PPI use are acknowledged by many scientific societies : treatment of the various forms and 
complications of gastroesophageal reflux disease, eradication of $\mathrm{H}$. pylori infection in combination with two or more antibiotics, short- and long-term therapy of $\mathrm{H}$. pylori-negative peptic ulcers, healing and prevention of NSAID/COXIB-associated gastric ulcers, co-therapy with endoscopic procedures to control upper digestive bleeding and medical treatment of Zollinger Ellison syndrome.

Expert Commentary:Despite the above well defined indications, however, the use of PPIs continues to grow every year in both western and eastern countries and the endless expansion of PPI market has created important problems for many regulatory authorities for two relevant features : the progressive increase of the costs of therapy and the greater potential harms for the patients. The major reasons for the misuse of PPIs are the prevention of gastro-duodenal ulcers in patients without risk factors and the stress ulcer prophylaxis in nonintensive care units, steroid therapy alone, anti-platelet or anti-coagulant treatment in patients without risk of gastric injury and the overtreatment of functional dyspepsia.

Key words : Proton pump inhibitors (PPIs); acid-related diseases; appropriate use; misuse; overprescription.

\section{Introduction}

The study of gastric acid secretion has attracted the interest of both physiologists and physicians for many years, because a great number of diseases of the upper gastrointestinal (GI) tract are related to dysfunctions in acid production. Many investigations have documented that gastric acid is produced by both resting and meal-stimulated parietal cells in the stomach, following neurocrine, paracrine and endocrine stimulation by various substances, such as acetylcholine, histamine or gastrin, which bind to their specific receptors placed on the baso-lateral membrane of the above cells (1). Thereafter, intracellular second messenger systems are activated leading to protein kinase formation and activation of $\mathrm{H}^{+} / \mathrm{K}^{+}$ ATPase enzyme (proton pump), which fuse with the secretory canaliculus of the parietal cell 
resulting in acid production, whereby intracellular hydrogen ions are exchanged for extracellular potassium ions (2). Once acid is produced, the lower luminal intragastric $\mathrm{pH}$ stimulates a feedback mechanism to maintain appropriate homeostatic control of acid secretion. This response is mediated primarily by paracrine release of somatostatin from gastric antral D cells, which inhibit G cell production of gastrin and enterochromaffin-like (ECL) formation of histamine in order to reverse the stimulus for acid secretion (3).

In relation to the above complex physiologic, mainly receptorial, mechanisms for acid secretion, drug research in this area has focused on the development of compounds able to increase the $\mathrm{pH}$ of gastric contents and the synthesis of many powerful inhibitors of gastric acid secretion has allowed us to control adequately acid-related diseases, thus minimizing the role of surgery in this field.

In fact, antisecretory therapy has advanced dramatically since the introduction of cimetidine in the mid-1970s and subsequently other molecules pertaining to this class of acidlowering agents have been added to the market, such as ranitidine, famotidine, nizatidine and roxatidine. All these drugs are able to block the histamine ${ }_{2}$ receptor on parietal cells, which is one of the three main pathways regulating gastric acid secretion, and this effect reduces hydrogen ion release into the stomach. However, $\mathrm{H}_{2}$-receptor antagonists $\left(\mathrm{H}_{2}\right.$-RAs) have several important limitations, in that they present a relatively short duration of action, which ranges approximately from 4 to 8 hours and therefore multiple daily doses of them are required, but even this repeated administration does not permit to obtain a complete control of acid production over the circadian cycle. Moreover, $\mathrm{H}_{2} \mathrm{RAs}$ have the relevant drawback of tolerance development, which generally occurs within two weeks of daily dosage. Finally, their antisecretory action is impaired in post-prandial periods, because meals are one of the most powerful stimulants of acid secretion, but this effect is mediated by gastrin, which is not blocked by $\mathrm{H}_{2} \mathrm{RAs}$.

The subsequent recognition that $\mathrm{H}^{+}-\mathrm{K}^{+}$-ATPase is the final step in the complex process of acid secretion led to the development of a new class of drugs, the proton pump inhibitors (PPIs), which are targeted at blocking specifically this enzyme. Conversely from $\mathrm{H}_{2} \mathrm{RAs}$, PPIs are able to control both basal and meal-stimulated acid secretion and produce a more complete and longer-lasting acid suppression, so that their pharmacodynamic effect is maintained for 16-18 hours (4), thus permitting the use of a single daily dose. Furthermore, they do not present the phenomenon of tolerance due to the lack of receptorial inhibition (5).

As result of the above-mentioned better pharmacological properties, PPIs have rapidly become the first-choice therapy in patients with acid-related disorders, replacing almost 
completely $\mathrm{H}_{2}$ RAs. The market of these drugs has progressively increased to the point that it is estimated that over 113 million PPI prescriptions are filled globally each year in the USA and this, together with over-the-counter (OTC) use, accounted for over 13,6 billion dollars worldwide in 2009 (6).

It must also be stressed that the introduction of generic PPIs into the market has contributed to increase remarkably PPI prescriptions (7). In addition, as PPIs are now available as OTC in many countries, patients can have free access to them and tend to take these drugs for long periods of time without seeking any medical attention (8).

However, the endless expansion of the PPI market has risen many concerns about the possible misuse of these drugs, which has created important problems for many regulatory authorities of various countries in both western and eastern countries. The two major issues are represented by the excessive costs of therapy for both patients and governments and the potential risk for iatrogenic harm due to adverse events related to these active and powerful drugs and to the unavoidable drug-to-drug interactions in patients taking multiple medications for concomitant diseases (9).

In relation to the above considerations, in the last years there has been a mounting number of reports linking the chronic use of PPIs to serious adverse reactions: gastric carcinoids, hip fractures, hypomagnesemia, nutritional deficiences, increased incidence of cardiovascular events, enteric infections, most notably Clostridium difficile diarrhea, community-acquired pneumonia, chronic kidney disease and dementia (10). Obviously, the above publications in prestigious international medical journals have contributed to produce alarmism in physicians and panic in many patients who are familiar with web navigation.

\section{Aims and methodology of the review}

In keeping with the above reflections, a review on the appropriate use of PPIs seems to be useful in order to emphasize the right indications clearly established for these medications and to highlight the most frequent reasons for their misuse in order to reduce their dangerous overutilization. To provide a summary of current information on the appropriate use and the misuse of PPIs, we took into account the guidelines published in english medical literature and the results of many clinical trials on the treatment of upper digestive disorders and the related meta-analyses and systematic reviews. In order to identify relevant studies, a computerized (Medline) and manual literature search was performed during the period from 1977 to the end of 2017, with particular focus on the past 15 years. We used the following terms : acid-related diseases, GERD or GORD, gastroesophageal reflux disease, acid reflux, NERD, non-erosive reflux disease, hypersensitive esophagus, reflux hypersensitivity, 
functional heartburn, esophageal pH-metry and impedance-pH testing, Barrett's esophagus, eosinophilic esophagitis and PPI responsive esophageal eosinophilia (PPI-REE), Helicobacter pylori infection, duodenal ulcer, H. pylori-negative duodenal ulcer, NSAID-induced ulcer, iatrogenic gastric ulcer, acid hypersecretory conditions, Zollinger Ellison syndrome (ZES), functional dyspepsia, NSAID-induced dyspepsia, stress ulcer, critically ill patients, intensive care units (ICUs), treatment and prevention of acid-related disorders, overutilization or overprescrition of PPIs, overuse or misuse of antisecretory drugs, costs of overprescribing PPIs. The above terms were used alone or in combination with the following ones : pathophysiology, treatment, management, PPIs, proton pump inhibitors, $\mathrm{H}_{2}$-blockers, $\mathrm{H}_{2}$ receptor antagonists, potential harms, adverse events, documented benefits, inappropriate prescription, cost-effectiveness, PPI expenditure. We critically reviewed all full-text papers and relevant abstracts published in english language. Moreover, the reference lists of the various contributions were searched to identify any additional studies that have been missed during the process.

\section{Approved indications for PPI therapy}

PPI therapy is indicated in the well defined clinical conditions reported in table I, according to the Food and Drug Administration (FDA) in USA (11) and the National Institute for Clinical Excellence (NICE) in UK (12).

\subsection{Healing of erosive esophagitis and its maintenance}

Excessive acid exposure in the distal part of the esophagus has been found in $75 \%$ $90 \%$ of patients with erosive esophagitis and, from a pathophysiological point of view, this is due to multiple factors : an impaired function of the lower esophageal sphincter which presents more inappropriate relaxations than normal, a reduced esophageal clearance of refluxed material, and a delayed gastric emptying in up to $40 \%$ of cases (13). Furthermore, the presence of hiatal hernia does favor reflux, even though this is only true for those longer than $2 \mathrm{~cm}$ (14). The final consequence of these multiple motor abnormalities is that more acid lies in the wrong place, that is in contact with esophageal mucosa and this phenomenon explains the excellent short-term results achieved with PPIs in healing mucosal lesions. In keeping with the meta-analysis by Chiba et al (15), about $85 \%$ of patients treated with PPIs had complete healing at 8 weeks compared with $52 \%$ of those receiving $\mathrm{H}_{2} \mathrm{RAs}$ and subsequent reviews were able to confirm these remarkable results (16), which are clearly linked to the greater and longer-lasting acid suppression provided by the inhibition of gastric proton pumps. 
However, GERD is a chronic relapsing disorder, because PPIs do not modify the multiple underlying motor alterations sustaining the disease pathogenesis and six months after stopping PPI therapy only $10 \%$ of patients with erosive esophagitis are still in remission (17). So, the prevention of recurrences represents a relevant aim of therapy in patients with erosive esophagitis. Vigneri et al (18) were the first Authors to show that once daily dose of omeprazole $20 \mathrm{mg}$ was able to maintain $80 \%$ of patients with erosive esophagitis free from mucosal lesions after 12 months of continuous treatment and this drug was significantly superior to both ranitidine and cisapride. The same result was later obtained with lansoprazole (15 and $30 \mathrm{mg}$ once daily), which proved to be capable of keeping about $80 \%$ of patients with erosive esophagitis in remission at 1 year (19). Therefore, PPIs are useful as maintenance therapy in order to prevent recurrences of both symptoms and mucosal lesions in patients with erosive esophagitis, particularly in those with the most severe forms (from B to $\mathrm{D}$, according to the Los Angeles classification).

\subsection{Non erosive reflux disease (NERD)}

In recent years we have realized that GERD is far more complex than previously thought as erosive esophagitis represents only about $30 \%$ of patients with GERD and the remaining 70\% pertain to the category of NERD, in whom no esophageal lesions are detected by endoscopy (20). Obviously, in this group symptom relief is the main goal of therapy and, accordingly, this aim combined with the improvement of quality of life has become the mirror of PPI effectiveness in the majority of GERD patients.

Furthermore, the modern application of 24-hour impedance-pH monitoring, which permits to distinguish acid from non-acidic refluxes (21), has allowed us to understand that NERD patients are greatly heterogeneous from a pathophysiological point of view and three distinct subgroups of them can be identified : a) true NERD with excess of acid in the esophagus b) hypersensitive esophagus to acid or non-acid refluxes with normal esophageal acid exposure and positive symptom-reflux association c) functional heartburn (FH), which is a category no more pertaining to the GERD realm, because there is neither excess of acid in their esophagus nor positive correlation between typical symptoms and reflux episodes of any type registered by impedance-pH testing (22).

In relation to the above stratification, it is clear that PPIs can be beneficial only in case of true NERD and hypersensitive esophagus to acid, whereas they do not work in patients with hypersensitive esophagus to non-acidic reflux and in those with $\mathrm{FH}$, where acid does not play any pathogenetic role. In fact, the event of PPI-refractory heartburn is more common in NERD than in erosive esophagitis and occurs in about $20 \%-30 \%$ of cases (23). In order to be 
sure that they are true PPI resistant patients, we have first to check whether they are compliant and adherent to treatment. There are several studies, indeed, showing that only $27 \%$ of patients assume these drugs correctly about 30 minutes before breakfast as once daily dose (24). As PPIs are able to control better daytime than nighttime acid secretion (25), in case of persistent and severe nocturnal symptoms, it is recommended to fraction the daily dose of them into two separate administrations, one before breakfast and the other before dinner, in order to achieve a more prolonged pharmacodynamic action up to the first hours of the night (26). Several reviews and meta-analyses (27) have confirmed that the various PPIs have almost equivalent efficacy in relieving satisfactorily symptoms of GERD in the short term (4-8 weeks). Obviously, the use of PPIs is inappropriate in patients with esophagus hypersensitive to non-acidic reflux and in $\mathrm{FH}$ and therefore these two last conditions require other therapeutic measures, mainly pain modulators (20).

Also NERD patients tend to relapse up to $75 \%$ of cases six months after stopping acute therapy (17) and therefore a long-term treatment is necessary even in them. This maintenance therapy for preventing symptom recurrence in NERD can be continuous in the the few cases with frequent recurrences or on demand in the majority of patients. On demand therapy is defined as the administration of drugs in response to symptoms with their discontinuation after symptoms are relieved and represents an attractive therapeutic option, because of cost-effectiveness and patient convenience. Many randomized clinical trials have shown the satisfactory success of the two above-mentioned long-term therapeutic approaches in NERD patients $(16,27)$.

\subsection{GERD with extra-esophageal symptoms}

Many papers have reported that GERD can present with extra-esophageal symptoms, such as hoarseness, chronic cough and asthma (28), although the causal relationship between these respiratory symptoms and reflux episodes remains highly controversial. Indeed, antireflux therapy with PPIs has been shown to be not superior to placebo in many of these patients, unless the relationship between GERD and atypical symptoms has been clearly proven by functional testing or concomitant typical symptoms are complained by patients. In fact, a recent review (29) suggests a therapeutic benefit by acid suppressing drugs in patients with chronic cough and this advocates a rigorous patients' selection that could allow the identification of a patients' subgroup likely to be PPI responsive. Conversely, no systematic reviews or meta-analyses showed any significant success of PPI therapy over placebo in patients with chronic laryngitis (30). Moreover, asthma and GERD can often coexist as 
abnormal reflux has been found in $40 \%-90 \%$ of cases (31). However, a Cochrane review (32) has denied any benefit from PPIs compared with placebo and only a more recent study has shown that asthma responds in patients with proven reflux and nocturnal symptoms (33). Nevertheless, in many cases the origin of extra-esophageal manifestations is multifactorial and an evident responsability of abnormal reflux cannot be documented even by means of modern sophisticated functional examinations (34). As the use of PPI doses higher than the standard ones and for longer duration than usual are suggested in these patients according to international guidelines, the efforts should be addressed to identify people with reasonable atypical symptoms-GERD association before embarking on a costly and prolonged PPI treatment.

\subsection{Esophageal strictures and Barrett's esophagus}

The incidence of esophageal strictures has decreased in the last two decades and this decline was associated with a marked increase in the use of PPIs. In fact, lansoprazole $30 \mathrm{mg}$ once daily resulted in fewer dilatations and a higher proportion of dysphagia-free patients than ranitidine $300 \mathrm{mg}$ b.i.d. (35).

Barrett's esophagus is the replacement of normal squamous epithelium of the distal part of the esophagus with columnar metaplasia and is the most important complication of GERD, because it is considered as a pre-cancerous lesion. It is associated with the maximal acid exposure compared with the other forms of this disease, so that the use of powerful antisecretory therapy with PPIs has a strong rationale. However, the results of medical treatment with these drugs has provided conflicting findings in terms of length reduction of intestinal metaplasia, whereas two retrospective $(36,37)$ and one prospective (38) studies have shown that PPIs may decrease its progression to adenocarcinoma, in that the rate of dysplasia development is reduced significantly in PPI-treated than in non-PPI-treated patients with Barrett's esophagus. This outcome is certainly more important than the simple reduction in length of intestinal metaplasia and supports the use of long-term PPIs in order to prevent the transformation of intestinal metaplasia into dysplasia and neoplasia. As further confirmation of this beneficial effect, it has been recently published a paper reporting the results of the eagerly awaited AspECT study, which has recruited 2257 patients with a median follow-up and treatment duration of 8-9 years (39). It showed that high-dose PPI and aspirin chemoprevention therapy, especially in combination, significantly and safely improved outcomes in terms of high-grade dysplasia, esophageal adenocarcinoma and all-cause mortality in patients with Barrett's esophagus.

\subsection{Eosinophilic esophagitis (EoE)}


EoE is a chronic immune-mediated inflammatory disorder, defined symptomatically by esophageal dysfunction and structurally by histological eosinophilic-predominant infiltrate (40). Esophageal eosinophilia is not limited to the distal part of the organ as in GERD and is frequently associated with serious motor alterations leading to dysphagia and food impaction (41). Although these patients can benefit from some antigen-free diets or topical corticosteroid therapy, some $30 \%-50 \%$ of them respond to twice daily PPI therapy (42). PPI responsive esophageal eosinophilia (PPI-REE) is considered a phenotype of EoE responding to PPIs, but recent studies have shown its similarity to GERD (43). It cannot be excluded, however, that the reason for the benefit of PPI therapy in this group of EoE patients can be due to effects other than the antisecretory action, namely their documented antiinflammatory activity (44). Indeed, both in vitro and in vivo investigations suggest that the anti-inflammatory effect of these drugs can be responsible for the improvement of the disease, being they capable of inhibiting the $\mathrm{Th}_{2}$ allergic pathway (45). Anyway, international guidelines indicate PPIs as first-line treatment of EoE and other effective alternatives, such as diet and topical steroids, should be used as second-line measures, because at present there are not criteria able to predict a good response to PPIs in part of these patients.

\subsection{Eradication of H. pylori infection}

A huge medical literature has clearly shown that chronic gastritis and most peptic ulcers have to be considered nowadays as infectious diseases, due to H. pylori which is a germ with an elective tropism for gastric mucosa (46). Indeed, many studies have confirmed that the eradication of this infection is able to cure chronic gastritis with good functional recovery of gastric acid secretion and to promote healing of ulcer with permanent disappearance of both recurrences and complications (47).

Different therapeutic schemes have been proposed over time and many of them include two antibiotics and a PPI given in two standard daily doses for 7-14 days (48). So, PPIs represent a key component of any currently adopted regimen for H. pylori eradication and many clinical trials have shown that it is possible to obtain rates of success as high as 80\%-90\% (49). As these rates tended to diminish in last years, mainly due to the development of clarithomycin resistance, recent Consensus statements suggest the use of quadruple regimens containing also bismuth as powerful drug against $H$. pylori (50). However, PPIs remain the mainstay of anti-Helicobacter therapy also in these more complex regimens.

\subsection{H. pylori-negative peptic ulcers}


Although it is well acknowledged that H. pylori is the cause of peptic ulcer, particularly duodenal ulcer, an increasing number of ulcer patients do not bear this germ (51). These $\mathrm{H}$. pylori-negative ulcers have been reported to be higher in USA and in Australia (20\%-50\%) than in Europe (52). The precise cause of these ulcers is unknown, but there is some evidence that a certain number of them is due to the unrevealed use of aspirin or NSAIDs (53). In the other cases the pathogenetic role of acid cannot be excluded, in that high basal and peak acid outputs have been found in duodenal ulcers relapsing despite H. pylori eradication (54). So, not only NSAID-induced ulcers can be treated with PPIs, but also idiopathic ulcers seems to benefit from acid-lowering drugs that need to be continued in the long term in order to maintain the ulcers healed (55).

\subsection{NSAID/COXIB-induced dyspepsia}

Dyspeptic symptoms can develop within the first few days of starting a NSAID/COXIB therapy and occur even with the first dose of these drugs (35). They can appear in the absence of any mucosal gastric lesion in about $15 \%$ of cases and the pathogenesis is not fully understood. However, a short course (4-8 weeks) of PPIs has been shown to control these symptoms adequately (56).

\subsection{Healing of NSAID-associated gastric ulcers and treatment of ulcer bleeding}

NSAIDs are among the most widely used classes of drugs and are very effective in controlling pain deriving by various inflammatory conditions. However, their success is associated with a very large number of adverse events in the gastrointestinal tract, particularly in the gastric mucosa. Indeed, an altered mucosa defense in the presence of a normal or decreased acid secretion seems to be the major culprit (1). In fact, despite lower acid profiles in the setting of gastric ulcers, a weak mucosa is fundamental to explain the effect of acid as secondary actor of the damaging process. Accordingly, it has been shown that the administration of NSAIDs, mainly diclofenac, for one month in patients with rheumatoid arthritis increases significantly intragastric acidity compared with baseline levels as likely result of blocking the antisecretory effect of prostaglandins, which are a well known target of NSAID therapy (57). In relation to this ancillary role of acid on a weakened mucosa, PPIs given for 4-8 weeks are able to provide ulcer healing, as shown in many randomized clinical trials (58).

The presence of multiple risk factors greatly increases the risk of ulcer complications, such as bleeding, as reported in table II. Among them, the most important are prior history of complicated ulcer and the age $>65$ years. The relevance of previous episodes of ulcer complications is obvious, while advancing age has been shown to increase the risk of 
bleeding by about 4\% per year, probably because of the presence of other factors and, among them, the role of concomitant $\mathrm{H}$. pylori infection, which is frequently found in elderly people, cannot be dismissed (35). So, the eradication of the germ should be pursued and can be particularly beneficial when starting treatment with NSAIDs or aspirin in patients with history of prior ulcer (59).

Although endoscopy remains the most useful therapeutic intervention to stop bleeding, the concomitant use of PPIs, possibly given both orally or intravenously before endoscopy, has the advantage of downgrading the stigmata of recent haemorrhage, thereby reducing the need for endoscopic hemostatic procedures, as confirmed by several meta-analyses (60). All the randomized controlled trials have shown that PPIs are much more effective than $\mathrm{H}_{2} \mathrm{RAs}$ in both treating and preventing gastroduodenal lesions (61), due to their greater degree and longer-lasting duration of acid inhibition. In fact, the objective of medical therapy for bleeding ulcers is to maintain a high intragastric $\mathrm{pH}$ ( $>6$ units) in order to promote platelet aggregation as well as the formation and stability of the clot inside the stomach (62), in that it is well known that platelet function is impaired at low $\mathrm{pH}$ values and pepsin promotes clot lysis below pH 5.0 (63).

Current guidelines (64) recommend the adoption of an intravenous bolus followed by a continuous infusion of PPIs, but a recent meta-analysis (65) showed that the efficacy of continuous or intermittent infusion does not differ. However, the Peptic Ulcer Bleed study, involving 91 hospital emergency departments of 16 countries, showed that high-dose intravenous esomeprazole ( $80 \mathrm{mg}$ in bolus followed by $8 \mathrm{mg} / \mathrm{h}$ infusion for 72 hours), given after successful endoscopic therapy in high-risk patients, reduced recurrent bleeding and kept a clinical benefit up to 30 days, while patients continued to take also orally esomeprazole 40 mg once daily (66).

\subsection{Prophylaxis of NSAID-associated gastric ulcer}

NSAIDs and aspirin are often prescribed chronically, especially in elderly subjects, for various important reasons, the latter drug mainly to prevent cerebral and cardiovascular ischemic events. Although they are very effective medications in this field, there is a great risk of complication development, particularly in the upper digestive tract. This risk is augmented in those patients bearing the already mentioned predisposing conditions listed in table II.

It must also be stressed that, although COX-2 selective NSAIDs (COXIBS) present a better safety profile than the traditional non-selective ones, they reduce the risk of gastric lesions by only $50 \%$ (67) and therefore the association of them with a PPI can be necessary, 
particularly in patients with the well known above risk factors in order to prevent ulcer formation and its complications.

International guidelines recommend the use of continuous PPI co-therapy in order to avoid gastric ulcer formation and gastrointestinal bleeding occurrence on the basis of the positive results of many trials showing that the combination of PPIs and NSAIDs or aspirin decrease the number of ulcers (68) and the recurrence of ulcer bleeding also in those taking the apparently safe low-dose aspirin (69).

\subsection{Treatment of Zollinger-Ellison syndrome}

This is the best characterized acid hypersecretory disorder and is caused by the tumor gastrinoma producing a large amount of gastrin, which in turn stimulates a remarkable acid hypersecretion (70). PPIs are the drug of choice for the medical treatment of ZES, but relatively high doses (3-4 times the standard dose) are required in comparison with those usually adopted for the other acid-related conditions (71). In fact, it is important that acid secretion is reduced below $10 \mathrm{mEq} / \mathrm{h}$ or below $5 \mathrm{mEq} / \mathrm{h}$ in post-surgical stomach to avoid ulcer recurrence and complications (72).

In patients who have undergone successful gastrinoma resection, PPIs may be still required because a hypersecretory status is kept in at least half of them (72). When surgical tumor removal is not possible, antisecretory therapy must be continued indefinitely.

\subsection{Prophylaxis of stress ulcers in critically ill patients}

Stress ulcer is an acute condition occurring in patients admitted in intensive care units (ICUs) and is commonly located in the proximal stomach. It seems to be the result of an ischemic damage reducing the mucosal defense, thereby acid acts only as a secondary factor. Current guidelines approve the use of PPIs as stress ulcer prophylaxis (SUP) in ICU patients at high risk of gastrointestinal bleeding, such as those who require mechanical ventilation for more than 48 hours or those with coagulopathy, who have an OR of 15.6 and 4.3, respectively (73). Several large meta-analyses found that the risk of bleeding in ICUs is reduced by about $60 \%$ in patients receiving SUP compared with those treated with placebo or no prophylaxis $(74,75)$. Therefore, SUP has become the standard of care in the ICUs, sometimes irrespective of the presence of risk factors.

\subsection{Functional dyspepsia}

Dyspepsia is a common condition seen in daily clinical practice of both general practitioners and gastroenterologists. It is characterized by one or more symptoms referred 
to the central upper part of the abdomen and, when endoscopy is negative and the intake of NSAIDs or aspirin has been excluded, the diagnosis of functional dyspepsia (FD) can be made. According to Rome IV criteria for functional gastrointestinal disorders (76), FD is subdivided into epigastric pain syndrome (EPS), including symptoms such as epigastric pain or burning, and post-prandial distress syndrome (PDS), including other symptoms such as epigastric fullness and early satiation. However, it is not rare that the symptoms of the two forms overlap.

Although acid hypersecretion does not exist in these patients (77), the lack of clear mechanisms originating the above symptoms lead physicians to adopt PPI therapy as the most frequent therapeutic attempt, particularly in cases of EPS $(9,35)$. So, the success of PPIs is scant and varies from $10 \%$ to about 30\%, according to several meta-analyses and the number needed-to-treat ranges from 10 to $15(78,79)$. There is no significant benefit from PPIs in PDS patients (35). So, considering the unsatisfactory results of PPIs in FD and the absence of other successful medications, a short-term, low-dose PPI treatment can be suggested in these patients, providing that there is a regular re-evaluation by physicians in order to avoid chronic treatment (80).

\section{Misuse of PPIs}

There is no doubt that PPI therapy has revolutionized our approach to the management of the above-mentioned acid-related conditions and the results have been very good and significantly superior to those achieved with $\mathrm{H}_{2} \mathrm{RAs}$.

Nevertheless, PPI use continues to grow every year in almost all western and eastern countries (81) and this calls into question the role of their misuse in both hospitals and primary care setting. In fact, it has been calculated that more than $50 \%$ of PPIs are prescribed inappropriately in general medical wards and in general practitioners' practice (9) and these rates are worrying, because they suggest that PPI use is often devoted to indications different from those recommended by expert consensus statements.

\subsection{Misuse of PPIs in the hospital setting}

The main reasons for PPI misuse in both hospital and primary care settings are reported in table III. Several investigations have analysed the rate of PPI overprescription in hospitalized patients. Reid et al (82) performed a retrospective review from two databases of university-affiliated hospitals in Colorado, taking into consideration randomly selected adult patients aged 18 to 90 years admitted for medical services. Appropriateness of PPI therapy was determined using International Classification of Diseases, 9th revision (ICD-9) codes. 
They found that $40 \%$ of patients in one databse and $14 \%$ of those in the other were prescribed PPIs during hospitalization. Adjusting data according to possible administrative errors, resulted that $>50 \%$ of cases failed to meet proper indications for PPI therapy. So, it is evident from this study that there is overuse of PPIs in US hospitalized patients, although the Authors recognized important limitations in their analysis. A further study conducted by Thomas et al (83) evaluated appropriate initiation of PPIs during hospitalization using again ICD-9 codes. PPI therapy was only considered appropriate if the patient was taking PPIs prior to hospitalization or if its use was consistent with diagnoses. A total of 20,197 (68.8\%) patients were categorized as inappropriate users of PPI therapy. Moreover, when comparing critically ill patients versus medical patients, there was no statistically significant difference found with respect to inappropriate use (68.7\% and 68.9\%, respectively). Evaluation of PPI misuse was continued over 4 years, although it decreased slightly during this time. However, there was no significant difference over time. Similarly to the already mentioned study by Reid et al, the accuracy of results may have been limited by the retrospective design of the investigation. However, the findings they reported are worrying and cannot be overlooked.

Gupta et al (84) evaluated retrospectively the inappropriate prescription of acid suppressive therapy, mainly PPIs, during hospital admission and at hospital discharge over a 3 month period in a sample of 279 patients. They found that an appropriate use of PPIs according to current guidelines and FDA approval was identified only in $27 \%$ of patients who were prescribed acid suppressant drugs upon admission to hospital and 69\% of those receiving inappropriate therapy were discharged with it. The most common reasons for this uncorrect prescription were SUP in low risk patients and ulcer prophylaxis in patients taking steroids alone or anti-coagulants without any risk factor. Despite the usual limitation of a retrospective study, the high rate of inappropriate PPI continuation upon discharge must be emphasized, because this illustrates the need for more thorough medication reconciliation prior to discharge.

Heidelbaugh et al (85) conducted a retrospective chart review over a 4-month period at a large university hospital on adult non-ICU patients to assess the practice of prescribing SUP. They found that of the 391 patient admissions prescribed SUP with almost exclusively PPIs, no patient met the criteria for SUP according to the guidelines and $54 \%$ of them were discharged on a prescription for acid suppressant therapy.

Finally, Zink et al (86) performed a retrospective chart review of general medical patients admitted to a teaching hospital over a 6-month period. Patients received an $\mathrm{H}_{2} \mathrm{RA}$ or a PPI. Appropriate indications were defined as an FDA-approved use or an indication 
supported by medical literature. Out of 324 patients included in the review, 128 (40\%) were initiated on acid suppressant therapy with an appropriate indication, whereas 196 (60\%) were inappropriate and $34 \%$ of them were discharged on the medication.

Overall, many studies have demonstrated high rates of inappropriate use of PPIs in the inpatient setting. All these studies are limited by their retrospective nature, but they confirm that the phenomenon is highly represented and the continuation of PPI therapy upon admission remains frequent, so this wrong behaviour must be discouraged in order to decrease costs and potential for serious adverse events and drug-to-drug interactions. The most common reason for this PPI misuse is SUP in low-risk patients, but ulcer prophylaxis in patients taking steroids alone or anti-coagulants without risk factors are also frequent inappropriate indications.

\subsection{Misuse of PPIs in the primary care setting}

In the primary care setting, the continuation of PPI intake after patients' discharge from hospitals and the absence of doctors' periodic review in subjects taking PPIs chronically are the main causes of inappropriate use of these drugs $(9,87)$. Rather surprisingly, too often PPIs are perceived by doctors themselves as a harmless and relatively inexpensive remedy for any digestive trouble which a patient has to encounter throughout her/his life. Indeed, we have above stressed that the quantity of patients who start PPIs after hospital admission is very high and the reasons for this therapy are often inconsistent and not related at all to a real pathogenetic role of acid. Interestingly, one study (88) has shown that the strongest factor associated with the inappropriate continuation of PPIs after hospital discharge was the intake of these drugs already before hospitalization and the lack of any critical reflection on their use during the inpatient stay, so that hospital doctors continue to prescribe these drugs at the discharge and general practitioners think that the prescription is correct and must be pursued in the long term.

FD represents another clinical area at great risk of PPI overpresciption, particularly in the long term and in those with the PDS variant. It is well known that many general practitioners prescribe these drugs to their patients indefinitely without any periodic reevaluation of whether there is really a continuing need to take this therapy or it is possible to reduce the dosage or to stop them at all, in order to avoid an unuseful overprescription. Moreover, functional dyspeptic symptoms are frequently associated with functional heartburn (89) or irritable bowel syndrome (90) and physicians must evaluate accurately the existence of these possible combinations, because they are predictive of poor response to PPI therapy (91). 
In patients taking steroids alone for whatever clinical condition, mucosal protection with a PPI is not routinely indicated $(35,92)$, unless they are associated with concomitant NSAID drugs. Indeed, several studies $(93,94)$ failed to demonstrate an association between corticosteroid use and risk of ulcer formation and its complications after adjustment for confounding factors. A meta-analysis also failed to show any significant risk for gastric and duodenal ulcers in patients receiving corticosteroid treatment compared to controls (92) and a systematic review of available meta-analyses in this field confirmed the same conclusion (95). In summary, no evidence currently supports the prophylactic use of PPIs in patients taking corticosteroids alone in order to prevent the development of peptic ulcers in the gastroduodenal tract.

NSAID/ASA and COXIB use in patients younger than 65 years and without other already-mentioned risk factors, gastroprotection with PPIs is not advised. In fact, Laine et al (96) found that older age increases the risk of developing a clinical gastrointestinal adverse event in individuals taking COXIBs or non-selective NSAIDs by stratifying 8,076 patients with rheumatoid arthritis into three subgroups of different age. In particular, the number needed to treat (NNT) to produce an adverse reaction was 10 in patients $>75$ years old, 25 in patients 65-74 years and 66 in those $<65$ years.

More recently, the synergistic role of NSAIDS and selective serotonin re-uptake inhibitors (SSRIs) has been highlighted, because it seems that there is a marked decrease in serotonin platelet content with consequent impairment of their aggregation in response to injury and the consequent prolongation of bleeding time (97). So, the association of NSAIDs and SSRIs can alter the hemostatic process and promote more severe bleeding and thus should be avoided; if this is not possible, the adoption of PPI therapy from the beginning of this combination treatment is highly recommended.

Also the administration of anti-coagulant compounds, such a slow molecular weight heparin or warfarin, in subjects without any risk factor is not an indication to associate PPI therapy, because these drugs are not directly gastrotoxic (9). A recent paper (98) has clearly demonstrated that PPI co-therapy had no significant protective effect for warfarin patients not using concomitant anti-platelet drugs or NSAIDs compared with those not taking PPIs, thus confirming that only the combination with gastrotoxic drugs enhances the rate of gastrointestinal bleeding and the need for hospitalization and then it requires PPI protection. When acenocoumarol is used as anti-coagulant, some caution is needed when prescribing PPIs (in particular omeprazole, esomeprazole and lansoprazole), because of potential overcoagulation most likely due to facilitated gastric absorption of warfarin (99), whereas no 
demonstrated interaction exists between PPIs and the novel oral anti-coagulants (35). Also ticlopidine or clopidogrel given alone in patients without risk factors do not require PPI use, which is mandatory only when the latter is associated with aspirin in the secondary prevention of cardiac ischemic attacks, because the combination of the two anti-platelet drugs is associated with serious harm of gastrointestinal bleeding (100). PPI co-therapy is able to reduce the risk of GI bleeding in these patients, although the well known interaction between them and clopidogrel at the level of CYP 2C19 in the liver with consequent lesser anti-platelet effect (101). PPIs that lack inhibition of hepatic CYP 2C19 (i.e. pantoprazole or rabeprazole) might be preferred in clopidogrel users (102).

In patients with liver cirrhosis and portal hypertension there is no benefit from PPI use, which is frequently adopted in daily clinical practice to prevent bleeding from hypertensive gastropathy, because it has been shown that their circadian intragastric acidity is significantly lower than in normal subjects (103). More importantly, there is some evidence that PPI use could be associated with development of spontaneous bacterial peritonitis (104).

Some studies have also shown that both a low income and a low educational level are associated wiht prolonged utilization and misuse of PPIs (105) and therefore other nonmedical reasons sustain this phenomenon. In fact, a nationwide cohort study carried out in Denmark (106) on patients taking for the first time PPIs and followed up for 10 years showed that the long-term utilization of these antisecretory drugs was significantly associated with low salary and low education, after adjusting the analysis to avoid other confounding variables. Also another Canadian study confirmed that living in areas of low socio-economic status was a predictor of a high and often incorrect consumption of PPIs on a chronic basis (107).

\subsection{Consequences of PPI overuse/misuse.}

The above overuse/misuse of PPIs is obviously responsible for a relevant increase of the costs of PPI therapy, up to the point that 2 billion pounds are being spent unnecessarily on PPIs each year (108). In a US study (85), the estimated cost for 1,034 patient-years treated inappropriately with PPIs was 1,566,252 dollars, based on average wholesale price costs. We have already mentioned the study by Thomas et al (83), who reviewed the pharmacy costs over a 4-year period of more than 20,000 patients receiving PPI therapy inappropriately after hospital admission and found that the unuseful global expense was approximately 3,000,000 dollars.

Finally, the overprescription of PPIs, especially in the elderly, can induce the appearance of various adverse events, as reported in many recent alarming publications 
$(10,109)$. However, many of these studies are observational and retrospective and then must be interpreted with great caution. In reality, if PPI therapy has an appropriate indication, the adverse reactions are confined within 1\%-3\%, a level compatible with that of any active and effective pharmacological compound (35). Some of them are plausible and predictable, while others are idiosyncratic, unpredictable and rare. The most serious harms with chronic PPI administration are represented by hypomagnesemia, enteric infections, mainly those from Clostridium difficile in elderly people, and acute kidney disease, such as idiosyncratic interstitial nephritis.

\subsection{Discontinuing long-term PPI therapy}

For patients who are taking PPI therapy for unclear indications or symptoms where there is little indication for PPI use, the discontinuation of these drugs must be attempted. However, the sudden withdrawal of PPIs may cause a marked rise in gastric acid output, a phenomenon known as rebound gastric acid hypersecretion. As a consequence, this may induce or aggravate upper GI symptoms. Reimer et al (110) were the first to show that 40\% of healthy individuals who discontinued PPIs after 8 weeks of treatment reported dyspepsia in the 4 weeks following withdrawal. Niklasson et al (111) showed that symptoms on PPI discontinuation were significantly correlated with the degree of PPI-induced hypergastrinemia. In a study in which 78 patients not having a clear indication for PPI use were discontinued, nearly two/thirds developed upper GI symptoms within 6 months and $40 \%$ of them had endoscopic findings consistent with their symptoms, mostly mild esophagitis (112). So, it seems important to avoid the abrupt withdrawal of PPIs, particularly in chronic users, and adopt a strategy aimed at reducing the phenomenon of rebound acid hypersecretion. PPI deprescribing can be achieved by stepping down to a lower dose or to an intermittent/on demand regimen or by replacing PPIs with a less potent acid inhibitor, such as $\mathrm{H}_{2}$ receptor antagonists (113).

\subsection{Conclusions}

There is no doubt that PPIs are effective drugs in the management of acid-related diseases, both in the short- and in the long-term. They have to be prescribed for the following major and universally recognized clinical conditions : treatment of GERD in its various forms and complications, PPI-REE as particular subgroup of eosinophilic esophagitis, eradication of H. pylori infection in combination with antibiotics, H. pylori-negative peptic ulcers, healing and prophylaxis of NSAID-associated gastric ulcers, co-therapy with endoscopic procedures in bleeding upper digestive ulcers and the Zollinger Ellison syndrome. 
The respect of the above guidelines could permit to reduce the high rate of PPI misuse, which is associated with increased costs and higher-than-expected incidence of adverse events. The major reasons for the misuse of PPIs are the prevention of gastro-duodenal ulcers in patients without risk factors and the stress ulcer prophylaxis in non-intensive care units, steroid therapy alone, anti-platelet or anti-coagulant treatment in patients without risk of gastric injury and the overtreatment of functional dyspepsia.

\section{Expert commentary}

The advent of PPIs as the most powerful blockers of gastric acid secretion in the last thirty years has allowed us to control adequately acid-related diseases, thus minimizing the role of surgery in this field. The main indications for PPI use, as acknowledged by the most important gastroenterological scientific societies, are represented by the various forms and complications of gastroesophageal reflux disease, the eradication of $\mathrm{H}$. pylori infection in combination with antibiotics, the short- and long-term therapy of $H$. pylori-negative peptic ulcers, the healing and prevention of NSAID/COXIB-associated gastric ulcers, the co-therapy with endoscopic procedures to control upper digestive bleeding and the medical treatment of Zollinger-Ellison syndrome.

However, the market of PPIs continues to grow in both western and eastern countries and there is evidence in many international publications that these drugs are prescribed inappropriately in both hospital and ambulatory settings. The main reasons for the misuse of PPIs are their administration in patients taking steroids alone, SSRIs alone, NSAIDs/COXIBs without risk factors, ticlopidine or clopidogrel or anti-coagulants alone without risk factors, the stress ulcer prophylaxis in non-intensive care units, the overtreatment of functional dyspepsia, the improper prescription of PPIs during hospital stay and their continuation after hospital discharge.

The major consequences of the misuse of PPIs are the excessive costs of therapy for both patients and governements and the risk of adverse events and possible drug-to-drug interactions in patients taking multiple medications for concomitant diseases. So, adhering to evidence-based guidelines represents the only rational approach to an effective and safe PPI therapy.

In conclusion, international scientific societies and health care authorities should develop specific programs aimed at educating and guiding both primary care physicians and specialists to the definite indications for PPI therapy and to eliminate unnecessary PPI use, thus reducing superfluous government expenditures and potentially serious adverse events in patients. 


\section{5 year view}

It is not easy to predict the behaviour of PPI market in the near future, but the risk that it will expand further remains possible. This continuous growth can be favoured by the introduction of generic PPIs into the market of many western countries, because this phenomenon may help to increase remarkably the PPI prescriptions in terms of chronic treatments, unlicensed indications and therapeutic substitutions. In addition, PPIs are now available as OTC in many countries and therefore patients can have free access to them with the consequence of taking these powerful drugs for long periods of time without seeking any medical attention. It must be also highlighted that the overprescrition of PPIs, particularly in the elderly, can induce the development of various adverse events, as reported in many recent alarming publications.

In order to reverse this potentially dangerous and costly trend of PPI overprescrition, only evidence-based recommedations, which consider the balance between benefits and harms, should be followed in every-day clinical practice. Therefore, we believe that gastroenterologists, who are the main class of doctors utilizing these drugs, together with the scientific societies and the regulatory authorities, should plan educational initiatives in order to guide both primary care physicians and hospitalists to the correct use of PPIs, according to the worldwide published gidelines.

\section{Key issues}

- PPIs are the best therapy for acid-related disorders

- The main indications for PPIs are represented by GERD, eradication of H. pylori infection in combination with antibiotics, H. pylori-negative peptic ulcers, healing and prevention of NSAID/COXIB-induced gastric ulcers and acid hypersecretory disorders, such as ZES

The market of PPIs continues to grow in many western and eastern countries

- Many international publications document that the inappropriate use of PPIs remains consistently high both in hospital and in primary care settings

- The main reasons for the misuse of PPIs are the prevention of gastro-duodenal ulcers in patients without risk factors, the stress ulcer prophylaxis in non-intensive care units, steroid therapy alone, anti-coagulant and anti-platelet treatment in patients without risk of gastric injury and the overtreatment of functional dyspepsia 
- The two main concerns pertaining to the misuse of PPIs are drug expenditure and risk of adverse events

- Education is the key to guide hospital doctors and general practitioners to the correct use of PPIs, according to worldwide published guidelines

\section{Funding}

This paper was not funded.

\section{Declaration of Interest}

The authors have no relevant affiliations or financial involvement with any organization or entity with a financial interest in or financial conflict with the subject matter or materials discussed in the manuscript. This includes employment, consultancies, honoraria, stock ownership or options, expert testimony, grants or patents received or pending, or royalties.

\section{Reviewer Disclosures}

Peer reviewers on this manuscript have no relevant financial or other relationships to disclose.

\section{References}

Papers of special note have been highlighted as:

* of interest

** of considerable interest

1. Schubert ML, Peura DA. Control of gastric acid secretion in health and disease. Gastroenterology 2004; 134:1842-1860. ** Complete and exhaustive review of gastric acid secretion in physiological and abnormal conditions.

2. Sachs G, Shin Gm, Howden CW. The clinical pharmacology of proton pump inhibitors. Aliment Pharmacol Ther 2006; 23(suppl): 2-8.

3. Vigneri S, Tonini M, Scarpignato $\mathrm{C}$, et al. Improving opportunities for effective management of gastro-oesophageal reflux disease. Dig Liver Dis 2001; 33:720-730.

4. Savarino V, Mela GS, Zentilin P, et al. Comparison of $24-\mathrm{h}$ control of gastric acidity by three different dosages of pantoprazole in patients with duodenal ulcer. Aliment Pharmacol Ther 1998; 12:1241-1247. 
5. Malesci A, Savarino V, Zentilin $P$, et al. Partial regression of Barrett's esophagus by long-term therapy with high-dose omeprazole. Gastrointest Endosc 1996; 44:700705.

6. Katz MH. Failing the acid test: benefits of proton pump inhibitors may not justify the risks for many users. Arch Intern Med 2010; 170:747-8.

7. Cammarota S, Bruzzese D, Sarnelli G, et al. Proton pump inhibitors prescribing following the introduction of generic drugs. Eur J Clin Invest 2012; 42:1068-1078.

8. Inadomi JM, Fendrick AM. PPI use in the OTC era: who to treat, with what, and for how long? Clin Gastroenterol Hepatol 2005; 3:208-215.

9. Savarino V, Dulbecco P, De Bortoli N, et al. The appropriate use of proton pump inhibitors (PPIs): need for a reappraisal. Eur J Intern Med 2017; 45:160-168. ** Complete and up-dated review on the appropriate indications of PPI therapy

10. Savarino V, Dulbecco P, Savarino E. Are proton pump inhibitors really so dangerous? Dig Liver Dis 2016; 48:851-859. ** Up-dated review on the mounting number of published adverse events as result of long-term PPI administration

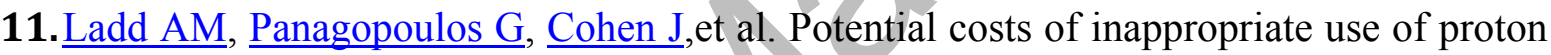
pump inhibitors. Am J Med Sci 2014; 347:446-451.

12. Batuwitage BT, Kingham JG, Morgan NE et al. Inappropriate prescribing of proton pump inhibitors in primary care. Postgrad Med J 2007; 83:66-68.

13. Savarino E, Gemignani L, Pohl D, et al. Oesophageal motility and bolus transit abnormalities increase in parallel with the severity of gastro-oesophageal reflux disease. Aliment Pharmacol Ther 2011; 34:476-486.

14. Frazzoni M, De Micheli E, Grisendi A, et al. Hiatal hernia is the key factor determining the lansoprazole dosage required for effective intra-oesophageal acid suppression. Aliment Pharmacol Ther 2002; 16:881-886.

15. Chiba N, De Gara CJ, Wilkinson JM, et al. Speed of healing and symptom relief in grade II to IV gastroesophageal reflux disease: a meta-analysis. Gastroenterology 1997; 112:1798-1810. * Important meta-analysis of the efficacy of PPI therapy in healing erosive esophagitis

16. Savarino V, Di Mario F, Scarpignato C. Proton pump inhibitor in GORD: An overview of their pharmacology, efficacy and safety. Pharmacol Res 2009; 59: 135-153 ** Exhaustive and up-dated review of PPI efficacy and safety in the treatment of GERD 
17. Carlsson R, Dent J, Watts R, Riley S, et al. Gastro-oesophageal reflux disease in primary care: an international study of different treatment strategies with omeprazole. International GORD Study Group. Eur J Gastroenterol Hepatol 1998; 10:119-124.

18. Vigneri S, Termini R, Leandro G, et al. A comparison of five maintenance therapies for reflux esophagitis. N Engl J Med 1995; 333:1106-1110

19. Labenz J, Armstrong D, Leodolter A, et al. Management of reflux esophagitis: does the choice of proton pump inhibitor matter? Int J Clin Pract 2015; 69:796-801.

20.Savarino E, Zentilin P, Savarino V. NERD: an umbrella term including heterogeneous subpopulations. Nat Rev Gastroenterol Hepatol 2013; 10:371-380. ** Complete review on the pathophysiological heterogeneity of patients with NERD

21.Zentilin P, Dulbecco P, Savarino E, et al. Combined multichannel intraluminal impedance and pH-metry: a novel technique to improve detection of gastro-oesophageal reflux literature review. Dig Liver Dis 2004; 36:565-569.

22. Savarino E, Bredenoord AJ, Fox M, et al; International Working Group for Disorders of Gastrointestinal Motility and Function. Expert consensus document: Advances in the physiological assessment and diagnosis of GERD. Nat Rev Gastroenterol Hepatol 2017; 14:665-676. ** Important and up-dated Consensus document on the modern diagnosis of GERD

23. Roman S, Gyawali CP, Savarino E, et al. Ambulatory reflux monitoring for diagnosis of gastro-esophageal reflux disease: Update of the Porto consensus and recommendations from an international consensus group. Neurogastroenterol Motil 2017; 29:1-15. ** Recent up-date of expert Consensus on the use of functional testing in the diagnosis of GERD

24. Gunaratnam NT, Jessup TP, Inadomi $\amalg$, et al. Sub-optimal proton pump inhibitor dosing is prevalent in patients with poorly controlled gastro-oesophageal reflux disease. Aliment Pharmacol Ther 2006; 23:1473-1477.

25. Savarino V, Mela GS, Zentilin P, et al. Antisecretory effects of three omeprazole regimens for maintenance treatment in duodenal ulcer. Dig Dis Sci 1994; 39:1473-1482.

26. Savarino V, Mela GS, Zentilin P, et al. Variability in individual response to various doses of omeprazole. Implications for antiulcer therapy. Dig Dis Sci 1994; 39:161-168.

27.van Pinxteren B, Numans ME, Bonis PA, et al. Shot-term treatment with proton pump inhibitors, $\mathrm{H}_{2}$-receptor antagonists and prokinetics for gastro-esophageal reflux disease-like symptoms and endoscopy-negative reflux disease. Cochrane 
Database Syst Rev 2006:3. CD002095. * Relevant meta-analysis of the efficacy of antisecretory drugs in the treatment of NERD

28. Ates F, Vaezi MF. Approach to the patient with presumed extra-esophageal GERD. Best Pract Res Clin Gastroenterol 2013 ; 27:415-431.

29. Kahrilas PJ, Howden CW, Hughes N, et al. Response of chronic cough to acidsuppressive therapy in patients with gastroesophageal reflux disease. Chest 2013; 143:605-612.

30. Gatta L, Vaira D, Sorrenti G, et al. Meta-analysis: the efficacy of proton pump inhibitors for laryngeal symptoms attributed to gastro-oesophageal reflux disease. Aliment Pharmacol Ther 2007; 25:385-392.

31. Scarpignato C. Pharmacological bases of the medical treatment of gastroesophageal reflux disease. Dig Dis 1988; 6:117-148.

32. Gibson PG, Henry RL, Coughlan JL. Gastro-oesophageal reflux treatment for asthma in adults and children. Cochrane Database Syst Rev 2003;(2):CD001496.

33. Kiljander TO, Harding SM, Field SK, et al. Effects of esomeprazole $40 \mathrm{mg}$ twice daily on asthma: a randomized placebo-controlled trial. Am J Respir Crit Care Med 2006; 173:1091-1097.

34.de Bortoli N, Nacci A, Savarino E, et al. How many cases of laryngopharyngeal reflux suspected by laryngoscopy are gastroesophageal reflux disease-related? World J Gastroenterol 2012; 18:4363-4370.

35. Scarpignato C, Gatta L, Zullo A, et al; SIF-AIGO-FIMMG Group; Italian Society of Pharmacology, the Italian Association of Hospital Gastroenterologists, and the Italian Federation of General Practitioners. Effective and safe proton pump inhibitor therapy in acid-related diseases - A position paper addressing benefits and potential harms of acid suppression. BMC Medicine 2016; 14:179-214.

Important and thoughtful position paper on the appropriate use and the safety of PPI therapy

36.El-Serag HB, Aguirre TV, Davis S, et al. Proton pump inhibitors are associated with reduced incidence of dysplasia in Barrett's esophagus. Am J Gastroenterol 2004; 99:1877-1883.

37.Hillman LC, Chiragakis L, Shadbolt B, et al. Proton-pump inhibitor therapy and the development of dysplasia in patients with Barrett's oesophagus. Med J Aust 2004; 180:387-391. 
38. Kastelein F, Spaander MC, Steyerberg EW, et al; ProBar Study Group. Proton pump inhibitors reduce the risk of neoplastic progression in patients with Barrett's esophagus. Clin Gastroenterol Hepatol 2013; 11:382-388. * The only prospective study on the usefulness of PPIs in reducing the rate of dysplasia in Barrett's esophagus

39. Jankowski JAZ, de Caestecker J, Love SB, et al. Esomeprazole and aspirin in Barrett's oesophagus (AspECT): a randomised factorial trial. Lancet 2018; 392:400-408. ** Very important and large prospective study on the role of chemopreventive therapy for improving the outcome of patients with Barrett's esophagus

40. Roman S, Savarino E, Savarino V, et al. Eosinophilic oesophagitis: from physiopathology to treatment. Dig Liver Dis 2013; 45:871-878.

41. Gyawali CP, Roman S, Bredenoord AJ, et al; International GERD Consensus Working Group. Classification of esophageal motor findings in gastro-esophageal reflux disease: Conclusions from an international consensus group. Neurogastroenterol Motil 2017; 29. doi: 10.1111/nmo.13104** Up-dated classification of esophageal motor disorders according to an international consensus group

42. Molina-Infante J, Bredenoord AJ, Cheng E, et al; PPI-REE Task Force of the European Society of Eosinophilic Oesophagitis (EUREOS). Proton pump inhibitor-responsive oesophageal eosinophilia: an entity challenging current diagnostic criteria for eosinophilic oesophagitis. Gut 2016; 65:524-531. ** Up-dated document of an european group working on esophageal eosinophilia

43. Savarino EV, Tolone $\mathrm{S}$, Bartolo $\mathrm{O}$, et al. The GerdQ questionnaire and high resolution manometry support the hypothesis that proton pump inhibitor-responsive oesophageal eosinophilia is a GERD-related phenomenon. Aliment Pharmacol Ther 2016; 44:522530.

44. Kedika RR, Souza RF, Spechler SI. Potential anti-inflammatory effects of proton pump inhibitors: a review and discussion of the clinical implications. Dig Dis Sci. 2009; 54:2312-2317.

45.de Bortoli N, Penagini R, Savarino E, et al. Eosinophilic esophagitis: Update in diagnosis and management. Position paper by the Italian Society of Gastroenterology and Gastrointestinal Endoscopy (SIGE). Dig Liver Dis 2017; 49:254-260. ** Position paper of the Italian Society of Gastroenterology on the diagnosis and management esophageal eosinophilia 
46.Zagari RM, Romano M, Ojetti V, et al. Guidelines for the management of Helicobacter pylori infection in Italy: The III Working Group Consensus Report 2015. Dig Liver Dis 2015; 47:903-912.

47.Graham DY, Lew GM, Klein PD, et al. Effect of treatment of Helicobacter pylori infection on the long-term recurrence of gastric or duodenal ulcer. A randomized, controlled study. Ann Intern Med 1992; 116:705-708.

48. Nyssen OP, McNicholl AG, Megraud F, et al. Sequential versus standard triple fîrst-line therapy for Helicobacter pylori eradication. Cochrane Database Syst Rev 2016; CD009034.

49. Ford A, Delaney B, Forman D, et al. Eradication therapy for peptic ulcer disease in Helicobacter pylori positive patients. Cochrane Database Syst Rev 2004; CD003840.

50. Fallone CA, Chiba N, van Zanten SV, et al. The Toronto Consensus for the Treatment of Helicobacter pylori Infection in Adults. Gastroenterology 2016; 151:51-69.e14.

51. Gisbert JP, Calvet X. Review article: Helicobacter pylori-negative duodenal ulcer disease. Aliment Pharmacol Ther 2009; 30:791-815.

52. Freston JW. Review article: role of proton pump inhibitors in non-H. pylori-related ulcers. Aliment Pharmacol Ther 2001; 15 Suppl 2:2-5.

53. Bytzer P, Teglbjaerg PS; Danish Ulcer Study Group. Helicobacter pylori-negative duodenal ulcers: prevalence, clinical characteristics, and prognosis--results from a randomized trial with 2-year follow-up. Am J Gastroenterol 2001; 96:1409-1416.

54.Harris AW, Gummett PA, Phull PS, et al. Recurrence of duodenal ulcer after Helicobacter pylori eradication is related to high acid output. Aliment Pharmacol Ther 1997; 11:331-334.

55. Howden CW, Leontiadis GI. Current indications for acid suppressants in Helicobacter pylori -negative ulcer disease. Best Pract Res Clin Gastroenterol 2001; 15:401-412.

56. Moayyedi P, Delaney BC, Vakil N, et al. The efficacy of proton pump inhibitors in nonulcer dyspepsia: a systematic review and economic analysis. Gastroenterology 2004; 127:1329-1337.

57. Savarino V, Mela GS, Zentilin P, et al. Effect of one-month treatment with nonsteroidal antiinflammatory drugs (NSAIDs) on gastric $\mathrm{pH}$ of rheumatoid arthritis patients. Dig Dis Sci 1998; 43:459-463.

58. Salas M, Ward A, Caro J. Are proton pump inhibitors the first choice for acute treatment of gastric ulcers? A meta analysis of randomized clinical trials. BMC Gastroenterol $2002 ; 2: 17$. 
59. Malfertheiner P, Megraud F, O'Morain CA, et al; European Helicobacter and Microbiota Study Group and Consensus panel. Management of Helicobacter pylori infection-the Maastricht V/Florence Consensus Report. Gut. 2017; 66:6-30. ** Last Maastrict/Florence up-date on the management of patients with Helicobacter pylori infection.

60.Leontiadis GI, Sharma VK, Howden CW. Proton pump inhibitor treatment for acute peptic ulcer bleeding. Cochrane Database Syst Rev 2006:CD002094.

61.Zhang YS, Li Q, He BS, et al. Proton pump inhibitors therapy vs $\mathrm{H} 2$ receptor antagonists therapy for upper gastrointestinal bleeding after endoscopy: A meta-analysis. World J Gastroenterol 2015; 21:6341-6351.

62. Berstad A. Does profound acid inhibition improve haemostasis in peptic ulcer bleeding? Scand J Gastroenterol 1997; 32:396-398.

63. Patchett SE, Enright H, Afdhal N, et al. Clot lysis by gastric juice: an in vitro study. Gut $1989 ; 30: 1704-1707$.

64.Laine L, Jensen DM. Management of patients with ulcer bleeding. Am J Gastroenterol 2012; 107:345-360.

65. Sachar $\mathrm{H}$, Vaidya $\mathrm{K}$, Laine L. Intermittent vs continuous proton pump inhibitor therapy for high-risk bleeding ulcers: a systematic review and meta-analysis. IAMA Intern Med. 2014; 174:1755-1762.

66. Sung JJ, Barkun A, Kuipers EJ, et al; Peptic Ulcer Bleed Study Group. Intravenous esomeprazole for prevention of recurrent peptic ulcer bleeding: a randomized trial. Ann Intern Med 2009; 150:455-464.

67. Moore RA, Derry S, Phillips CJ, et al. Nonsteroidal anti-inflammatory drugs (NSAIDs), cyxlooxygenase-2 selective inhibitors (coxibs) and gastrointestinal harm: review of clinical trials and clinical practice. BMC Musculoskelet Disord 2006; 7:79.

68. Rostom A, Dube C, Wells G, et al. Prevention of NSAID-induced gastroduodenal ulcers. Cochrane Database Syst Rev 2002:CD002296.

69. Tran-Duy A, Vanmolkot FH, Joore MA, et al. Should patients prescribed long-term low-dose aspirin receive proton pump inhibitors? A systematic review and metaanalysis. Int J Clin Pract 2015; 69:1088-1111. * Important paper on the use of PPIs in patients taking long-term low-dose aspirin

70.Jensen RT, Niederle B, Mitry E, Ramage JK, et al; Frascati Consensus Conference; European Neuroendocrine Tumor Society. Gastrinoma (duodenal and pancreatic). Neuroendocrinology 2006; 84:173-182 
71. Jensen RT, Cadiot G, Brandi ML, et al; Barcelona Consensus Conference participants. ENETS Consensus Guidelines for the management of patients with digestive neuroendocrine neoplasms: functional pancreatic endocrine tumor syndromes. Neuroendocrinology 2012; 95:98-119. ** Consensus guidelines on the management of patients with Zollinger- Ellison syndrome

72. Wilcox CM, Hirschowitz BI. Treatment strategies for Zollinger-Ellison syndrome. Expert Opin Pharmacother 2009; 10:1145-1157

73. Bardou M, Quenot JP, Barkun A. Stress-related mucosal disease in the critically ill patient. Nat Rev Gastroenterol Hepatol 2015; 12:98-107.

74. Alhazzani W, Alenezi F, Jaeschke RZ, et al. Proton pump inhibitors versus histamine 2 $\underline{\text { receptor antagonists for stress ulcer prophylaxis in critically ill patients: a systematic }}$ review and meta-analysis. Crit Care Med 2013; 41:693-705.

75. Barkun AN, Bardou M, Pham CQ, et al. Proton pump inhibitors vs. histamine 2 receptor antagonists for stress-related mucosal bleeding prophylaxis in critically ill patients: a meta-analysis. Am J Gastroenterol 2012; 107:507-520.

76. Stanghellini V, Chan FK, Hasler WL, et al. Gastroduodenal Disorders. Gastroenterology 2016; 150:1380-1392. ** Important paper on the Rome IV criteria for gastroduodenal functional disorders

77. Savarino V, Mela GS, Zentilin P, et al. Circadian gastric acidity in Helicobacter pylori positive ulcer patients with and without gastric metaplasia in the duodenum. Gut 1996; 39:508-512.

78. Moayyedi P, Soo S, Deeks J, et al. Pharmacological interventions for non-ulcer dyspepsia. Cochrane Database Syst Rev 2006:CD001960.

79. Wang WH, Huang JQ, Zheng GF, et al. Effects of proton-pump inhibitors on functional dyspepsia: a meta-analysis of randomized placebo-controlled trials. Clin Gastroenterol Hepatol 2007; 5:178-185.

80. National Institute for Clinical Excellence. Management of dyspepsia in adults in primary care. August 2004, revised July 2005.

81.van Vliet EP, Otten HJ, Rudolphus A, et al. Inappropriate prescription of proton pump inhibitors on two pulmonary medicine wards. Eur J Gastroenterol Hepatol 2008; 20:608612.

82. Reid M, Keniston A, Heller JC, et al. Inapprpriate prescribing of proron pump inhibitors in hospitalized patients. J Hosp Med 2012; 7: 421-425. 
83.Thomas $\mathrm{L}^{1}$, Culley EI, Gladowski P, et al. Longitudinal analysis of the costs associated with inpatient initiation and subsequent outpatient continuation of proton pump inhibitor therapy for stress ulcer prophylaxis in a large managed care organization. J Manag Care Pharm. 2010; 16:122-129.

84. Gupta R, Garg P, Kottoor et al. Oversuse of acid suppression therapy in hospitalized patients. South Med J 2010; 103: 207-211.

85. Heidelbaugh JJ, Inadomi JM. Magnitude and economic impact of inappropriate use of stress ulcer prophylaxis in non-ICU hospitalized patients. Am J Gastroenterol 2006; 101:2200-2205.

86.Zink DA, Pohlman M, Barnes $M$, et al. Long-term use of acid suppression started inappropriately during hospitalization. Aliment Pharmacol Ther 2005; 21: 1203-1209.

87. Heidelbaugh JJ, Kim AH, Chang R, et al. Overutilization of proton-pump inhibitors: what the clinician needs to know. Therap Adv Gastroenterol 2012; 5:219-232.

88. Ahrens D, Behrens G, Himmel W, et al. Appropriateness of proton pump inhibitor recommendations at hospital discharge and continuation in primary care. Int J Clin Pract 2012; 66:767-773.

89. Savarino E, Pohl D, Zentilin P, et al. Functional heartburn has more in common with functional dyspepsia than with non-erosive reflux disease. Gut 2009; 58:1185-1191.

90.de Bortoli N, Frazzoni L, Savarino EV, et al. Functional Heartburn Overlaps With Irritable Bowel Syndrome More Often than GERD. Am J Gastroenterol 2016; 111:17111717.

91.Zerbib F, Belhocine K, Simon M, et al. Clinical, but not oesophageal pH-impedance, profiles predict response to proton pump inhibitors in gastro-oesophageal reflux disease. Gut 2012; 61:501-506.

92.Hernández-Díaz S, Rodríguez LA. Steroids and risk of upper gastrointestinal complications. Am J Epidemiol 2001; 153:1089-1093.

93.Luo JC, Chang FY, Lin HY, Lu RH, Lu CL, Chen CY, Lee SD. The potential risk factors leading to peptic ulcer formation in autoimmune disease patients receiving corticosteroid treatment. Aliment Pharmacol Ther 2002; 16:1241-1248.

94. Conn HO, Poynard T. Corticosteroids and peptic ulcer: meta-analysis of adverse events during steroid therapy. J Intern Med 1994; 236:619-632

95. Dorlo TP, Jager NG, Beijnen JH, Schellens JH. [Concomitant use of proton pump inhibitors and systemic corticosteroids]. Ned Tijdschr Geneeskd 2013; 157:A5540. 
96. Laine L, Bombardier C, Hawkey CJ, et al. Stratifying the risk of NSAID-related upper gastrointestinal clinical events: results of a double-blind outcomes study in patients with rheumatoid arthritis. Gastroenterology 2002; 123:1006-1012.

97. de Abajo FJ. Effects of selective serotonin reuptake inhibitors on platelet function: mechanisms, clinical outcomes and implications for use in elderly patients. Drugs Aging 2011; 28:345-367.

98. Ray $\mathrm{WA}^{1}$, Chung $\mathrm{CP}^{2}$, Murray $\mathrm{KT}^{3}$, et al. Association of Proton Pump Inhibitors With Reduced Risk of Warfarin-Related Serious Upper Gastrointestinal Bleeding. Gastroenterology 2016; 151:1105-1112.

99.Teichert M, van Noord C, Uitterlinden AG, et al. Proton pump inhibitors and the risk of overcoagulation during acenocoumarol maintenance treatment. Br J Hematol $2011 ; 153: 379-385$.

100. Lin $\mathrm{KJ}^{1}$, Hernández-Díaz $\mathrm{S}$, García Rodríguez LA. Acid suppressants reduce risk of gastrointestinal bleeding in patients on antithrombotic or anti-inflammatory therapy. Gastroenterology 2011;141:71-79.

101. Agewall S, Cattaneo M, Collet JP, et al. ESC Working Group on Cardiovascular Pharmacology and Drug Therapy and ESC Working Group on Thrombosis. Expert position paper on the use of proton pump inhibitors in patients with cardiovascular disease and antithrombotic therapy. Eur Heart J 2013; 34:1708-1713, 1713a-1713b.

102. Chen M, Wei JF, Xu YN, et al. A meta-analysis of impact of proton pump inhibitors on antiplatelet effect of clopidogrel. Cardiovasc Ther 2012; 30:e227-233.

103. Savarino V, Mela GS, Zentilin $P$, et al. Evaluation of 24-hour gastric acidity in patients with hepatic cirrhosis. J Hepatol 1996; 25:152-157.

104. Deshpande A, Pasupuleti V, Thota P, et al. Acid-suppressive therapy is associated with spontaneous bacterial peritonitis in cirrhotic patients: a meta-analysis. $\mathrm{J}$ Gastroenterol Hepatol 2013; 28:235-242 ** Important meta-analysis regarding the association between PPI use and spontaneous bacterial peritonitis in cirrhotic patients

105. van Boxel OS, Hagenaars MP, Smout AJ, et al. Socio-demographic factors influence chronic proton pump inhibitor use by a large population in the Netherlands. Aliment Pharmacol Ther 2009; 29:571-579.

106. Haastrup PF, Paulsen MS, Christensen RD, et al. Medical and non-medical predictors of initiating long-term use of proton pump inhibitors: a nationwide cohort 
study of first-time users during a 10-year period. Aliment Pharmacol Ther 2016; 44:7887.

107. Targownik LE, Metge C, Roos L, et al. The prevalence of and the clinical and demographic characteristics associated with high-intensity proton pump inhibitor use. Am J Gastroenterol 2007; 102:942-950.

108. Forgacs I, Loganayagam A. Overprescribing proton pump inhibitors. BMJ 2008; 336(7634):2-3.

109. Eusebi LH, Rabitti S, Artesiani ML, et al. Proton pump inhibitors: risks of long-term use. J Gastroenterol Hepatol 2017; 32: 1295-1302.

110. Reimer C, Søndergaard B, Hilsted L, Bytzer P. Proton-pump inhibitor therapy induces acid-related symptoms in healthy volunteers after withdrawal of therapy. Gastroenterology 2009; 137:80-87,

111. Niklasson A, Lindström L, Simrén M, et al. Dyspeptic symptom development after discontinuation of a proton pump inhibitor: a double-blind placebo-controlled trial. Am $\mathbf{J}$ Gastroenterol 2010; 105:1531-1537.

112. Reimer C, Bytzer P. Discontinuation of long-term proton pump inhibitor therapy in primary care patients: a randomized placebo-controlled trial in patients with symptom relapse. Eur J Gastroenterol Hepatol 2010; 22:1182-1188.

113. Targownik L. Discontinuing Long-Term PPI Therapy: Why, With Whom, and How? Am J Gastroenterol 2018; 113:519-528. ** Relevant paper of the problem of PPI discontinuation, particulalrly in long-term users 


\section{Table I. Internationally approved indications for PPI therapy}

- Healing of erosive esophagitis and its maintenance

- Non-erosive reflux disease (NERD) with excess of acid or acid hypersensitivity

- Gastroesophageal reflux disease (GERD) with ascertained extra-esophageal symptoms

- Complications of GERD, including esophageal strictures and Barrett's esophagus

- PPI-responsive esophageal eosinophilia

- Eradication of Helicobacter pylori infection in combination with antibiotics

- Treatment of H. pylori-negative peptic ulcers

- Symptomatic treatment of NSAID-induced dyspepsia

- Healing of NSAID-associated gastric ulcers and treatment of their bleeding

- Prophylaxis of NSAID-associated gastric ulcers

- $\quad$ Medical therapy of Zollinger Ellison syndrome (ZES)

- Prophylaxis of stress ulcers in critically ill patients, particularly those on prolonged mechanical ventilation and presence of coagulopathy 
- Short-term treatment of patients with functional dyspepsia, particularly those with the epigastric pain variant (EPS)

Table II. Risk factors indicating the need for appropriate prophylaxis with PPIs

- History of ulcer complications, particularly bleeding

- $\quad$ Age $>65$ years

- Prior ulcer

- NSAIDs/COXIBs at high doses or in combination with other gastrotoxic drugs or anti-coagulants (multiple NSAIDs/COXIBs, steroids, selective serotonin re-uptake inhibitors, warfarin)

Aspirin use, even at low dosage in elderly patients, or combined with other drugs (NSAIDS/COXIBs, steroids, anticoagulants, clopidogrel)

- Ticlopidine or clopidogrel use in patients at high risk (see above)

- Acute NSAID/COXIB use in patients taking chronically anti-coagulant or antiplatelet drugs

\section{Table III. Main causes of misuse of PPI therapy}

- Steroids intake alone

- SSRIs intake alone 
- $\quad$ NSAID/ASA/COXIB use in patients $<65$ yrs or without other risk factors

- Low molecular weight heparin or warfarin alone without risk factors

- Ticlopidine or clopidogrel alone without risk factors

- Stress ulcer prophylaxis in non-ICUs

- Wrong diagnosis of acid-related disorder

- Overtreatment of functional dyspepsia

- Patients with chronic liver disease and portal hypertension

- Improper prescription of PPIs during hospital stay

- Continuation of PPIs prescribed inappropriately upon hospital discharge

- Absence of regular doctors' re-evaluation of their patients assuming chronically PPI therapy 